\title{
Stereotactic body radiotherapy for stage I non-small-cell lung cancer using higher doses for larger tumors: results of the second study
}

Akifumi Miyakawa ${ }^{1 *}$ D, Yuta Shibamoto ${ }^{1}$, Fumiya Baba ${ }^{2}$, Yoshihiko Manabe ${ }^{1}$, Taro Murai ${ }^{1}$, Chikao Sugie ${ }^{1}$ Takeshi Yanagi ${ }^{1}$ and Taiki Takaoka ${ }^{1}$

\begin{abstract}
Background: Efficacy of stereotactic body radiotherapy (SBRT) in stage I non-small-cell lung cancer (NSCLC) has almost been established. In Japan, the protocol of 48 Gy in 4 fractions over 4 days has been most often employed, but higher doses may be necessary to control large tumors. Previously, we conducted a clinical study using SBRT for stage I NSCLC employing different doses depending on tumor diameter, which was closed in 2008. Thereafter, a new study employing higher doses has been conducted, which is reported here. The purpose of this study was to review the safety and effectiveness of the higher doses.
\end{abstract}

Methods: We escalated the total dose for the improvement of local control for large tumors. In this study, 71 patients underwent SBRT between December 2008 and April 2014. Isocenter doses of 48, 50, and 52 Gy were administered for tumors with a longest diameter of $<1.5 \mathrm{~cm}, 1.5-3 \mathrm{~cm}$, and $>3 \mathrm{~cm}$, respectively. It was recommended to cover $95 \%$ of the PTV with at least $90 \%$ of the isocenter dose, and in all but one cases, $95 \%$ of the PTV received at least $80 \%$ of the prescribed dose. Treatments were delivered in 4 fractions, giving 2 fractions per week. SBRT was performed with 6-MV photons using 4 non-coplanar and 3 coplanar beams.

Results: The median follow-up period was 44 months for all patients and 61 months for living patients. Overall survival (OS) was 65\%, progression-free survival (PFS) was 55\%, and cumulative incidence of local recurrence (LR) was 15\% at 5 years. The 5 -year OS was $69 \%$ for 57 stage IA patients and $53 \%$ for 14 stage IB patients $(p=0.44)$. The 5 -year PFS was 55 and $54 \%$, respectively $(p=0.98)$. The 5 -year cumulative incidence of LR was 11 and $31 \%$, respectively $(p=0.09)$. The cumulative incidence of Grade $\geq 2$ radiation pneumonitis was $25 \%$.

Conclusions: Our newer SBRT study yielded reasonable local control and overall survival and acceptable toxicity, but escalating the total dose did not lead to improved outcomes.

Trial registration: UMIN000027231, registered on 3 May 2017. Retrospectively registered.

Keywords: Non-small-cell lung cancer, Stereotactic body radiotherapy, Dose escalation, Overall survival, Toxicity

\footnotetext{
* Correspondence: amiyakawa21@triton.ocn.ne.jp

${ }^{1}$ Department of Radiology, Nagoya City University Graduate School of

Medical Sciences, 1 Kawasumi, Mizuho-cho, Mizuho-ku, Nagoya 467-8601,

Japan

Full list of author information is available at the end of the article
} 


\section{Background}

Stereotactic body radiotherapy (SBRT) is used for the treatment of primary cancer and oligometastatic disease $[1,2]$. In particular, the efficacy of SBRT in stage I nonsmall cell lung cancer (NSCLC) has been generally established [3-12]. In Japan, the protocol of 48 Gy in 4 fractions over 4 days is the most widely employed for both stage IA and IB NSCLC $[3,7,10]$. However, it was found that the outcomes of stage IB patients were worse than those of stage IA patients at the same dose [4, 7]. Therefore, we postulated that higher doses may be necessary to control larger tumors.

We evaluated the clinical outcomes of SBRT for stage I NSCLC employing different doses depending on tumor diameter in our first study $[13,14]$. Based on these results, we escalated the total dose in order to improve outcomes in a second study. In addition, we changed the dose calculation algorithm from pencil beam convolution to the analytical anisotropic algorithm (AAA), which is expected to improve dose distribution. We hypothesized that dose escalation using the AAA algorithm would be feasible and lead to improved outcome. Eight years have passed since we started the second protocol, so we analyzed the clinical outcomes of patients involved in the second study.

\section{Methods}

\section{Study design and eligibility criteria}

The study protocols were approved by the institutional review board (NCU-0803). Informed consent was obtained from all patients before SBRT. The present second study aimed to accrue 180 patients in accordance with the first study $[14,15]$; however, because many affiliated hospitals started SBRT for lung cancer during the last 10 years, patient accrual became much slower at our institution. So, we decided to perform an interim analysis for the second study, the results of which are reported here, in order to evaluate the adequacy of continuing the protocol.

Eligibility criteria were similar to the previous study: (1) histologically confirmed stage I NSCLC diagnosed according to the 7th TNM classification of lung cancer by the Union for International Cancer Control by chest and upper abdomen CT, brain MRI, and bone scintigraphy or FDG-PET; (2) greatest tumor diameter $\leq 5 \mathrm{~cm}$; (3) World Health Organization performance status (PS) $\leq 2$ or PS 3 when its cause was not a pulmonary disease; (4) no prior therapy and no concurrent malignancy; and (5) arterial oxygen pressure $\geq 60 \mathrm{mmHg}$, and forced expiratory volume in $1 \mathrm{~s} \geq 700 \mathrm{ml}$.

\section{Patient characteristics}

Seventy one patients underwent SBRT between December 2008 and April 2014. All patients completed the planned treatment. Patients were deemed medically inoperable when they had a poor pulmonary function (ratio of forced expiratory volume in $1 \mathrm{~s}$ to forced vital capacity $<60 \%$ and/or percent vital capacity $<75 \%$ ) or other debilitating conditions that precluded surgery. The patient and tumor characteristics are shown in Table 1. The tumor location was classified into central or peripheral in accordance with Radiation Therapy Oncology Group criteria [16].

\section{Treatment}

Our treatment methods were described in detail previously $[13,15]$. We used the BodyFIX system (Medical Intelligence, Schwabmuenchen, Germany) for patient immobilization. The visible gross tumor volume on CT during three phases (normal breathing and breath holding during the expiratory and inspiratory phases) was superimposed to represent the internal target volume (ITV). Breath-holding-phase CT images were used to ensure the range of tumor motion. During the first study, we had confirmed that a forced inspiration/expiration breath hold would not overestimate the tumor motion and therewith systematically overestimate the margins, by using fluoroscopy [17]. The planning target volume (PTV) margin for the ITV was $5 \mathrm{~mm}$ in the lateral and anteroposterior directions and $5-10 \mathrm{~mm}$ in the craniocaudal direction. Forward planning was performed using a 3-dimensional treatment planning system (Eclipse Version 7.5.14.3, Varian Medical Systems, Palo Alto, California, USA). Fixed 3 coplanar and 4 non-coplanar beams were used in all cases.

For verification of tumor positions, we used the simulator $\mathrm{CT}$ at the first and third treatments in addition to megavoltage portal imaging at every treatment

Table 1 Patients and tumor characteristics

\begin{tabular}{ll}
\hline Characteristics & \\
\hline Patient number & 71 \\
Age (years), range (median) & $55-89(77)$ \\
Gender, male/female & $51 / 20$ \\
Performance status (0/1/2) & $32 / 33 / 6$ \\
T stage (T1a/T1b/T2a) & $24 / 33 / 14$ \\
Total dose (48 Gy/50 Gy/52 Gy) & $6 / 51 / 14$ \\
Tumor location & \\
$\quad$ Center/periphery & $8 / 63$ \\
Operability & \\
Operable/inoperable & $24 / 47$ \\
Operable (T1a/T1b/T2a) & $12 / 8 / 4$ \\
Inoperable (T1a/T1b/T2a) & $12 / 25 / 10$ \\
Histology & \\
Adeno/squamous/NSCLC & $50 / 14 / 7$ \\
\hline Adeno adenocarcinoma, squamous squamous cell carcinoma, NSCLC
\end{tabular}

Adeno adenocarcinoma, squamous squamous cell carcinoma, NSCLC unclassified non-small-cell lung cancer 
throughout the study period. The patients underwent registration in the CT simulator room, and repositioning was performed whenever necessary. Then, they were carefully transferred to the linac room with a stretcher. SBRT was delivered by CLINAC 23EX (Varian Medical Systems, Palo Alto, California, USA) with 6-MV photon beams and it was delivered with 4 fractions. In principle, the respective fractions were delivered at intervals of $\geq 72 \mathrm{~h}$ to allow reoxygenation of hypoxic tumor cells [14], but owing to national holidays, patient schedule convenience, and machine availability, the actual overall treatment period was 8-20 days (median, 11 days). The total dose at the isocenter was increased to 48 Gy for tumors with a maximum diameter $<1.5 \mathrm{~cm}$ and $50 \mathrm{~Gy}$ for tumors of $1.5-3 \mathrm{~cm}$. For those $>3 \mathrm{~cm}$, the total dose remained at 52 Gy. The dose calculation algorithm was AAA.

It was recommended to cover $95 \%$ of the PTV with at least $90 \%$ of the isocenter dose, and, in all cases, $95 \%$ of the PTV received at least $80 \%$ of the prescribed dose. However, the dose was $79.2 \%$ in one case. Dose constraints for normal tissues were: (1) volume of the lung receiving $20 \mathrm{~Gy}, \leq 20 \%$; (2) $40 \mathrm{~Gy}$ for $<1 \mathrm{~cm}^{3}$ of the pulmonary artery and esophagus; (3) 36 Gy for $<10 \mathrm{~cm}^{3}$ of the stomach; and (4) maximum cord dose < $18 \mathrm{~Gy}$, in accordance with the first study $[13,14]$.

\section{Evaluation}

Chest and upper abdominal CT was performed at 2month intervals until 6 months, and every 2-4 months thereafter. FDG-PET was performed whenever necessary. Local recurrence was diagnosed using serial CT examinations combined with FDG-PET and/or biopsy, as described in detail previously [18]. Pleuritis carcinomatosa unaccompanied by local recurrence was regarded as distant metastasis. Toxicity was evaluated using the Common Terminology Criteria for Adverse Events version 4 . Follow-up after 5 years was conducted at the discretion of the attending radiation oncologist.

\section{Statistical analysis}

Overall survival (OS) and progression-free survival (PFS) were calculated from the start of SBRT using the Kaplan-Meier method. The log-rank test was used to compare these curves. A Fine and Gray competing-risks regression model was used to estimate and compare cumulating incidences of local recurrence (LR), thereby considering patient death as a competing risk. Incidence of complications was compared using Fisher exact test. All statistical analyses were carried out using $R$ version 2.13.0 for Windows (The R Foundation for Statistical Computing, Vienna, Austria). $p$-values of $<0.05$ were defined as significant.

\section{Results}

Efficacy

The median follow-up period was 44 months for all patients and 61 months for living patients. At 5 years, OS was $65 \%$, PFS was $55 \%$, and the cumulative incidence of LR was $15 \%$. Figs. 1a, 2a and 3a show OS, PFS, and cumulative incidence of LR in all patients, operable patients, and medically inoperable patients. The OS, PFS, and cumulative incidence of LR did not differ between the operable patients and inoperable patients (Table 2). Figs. $1 \mathrm{~b}, 2 \mathrm{~b}$ and $3 \mathrm{~b}$ show OS, PFS, and cumulative incidence of LR in patients treated with 48, 50, and $52 \mathrm{~Gy}$. The 3- and 5-year data in these patients are shown in Table 3. The OS, PFS, and cumulative incidence of LR did not differ among the 3 groups. The 5 -year OS was $69 \%$ for 57 stage IA patients and $53 \%$ for 14 stage IB patients $(p=0.44)$. The 5 -year PFS was 55 and $54 \%$, respectively $(p=0.98)$. The 5 -year cumulative incidence of LR was 11 and $31 \%$, respectively $(p=0.09)$.

\section{Toxicities}

The cumulative incidence of Grade $\geq 3$ radiation pneumonitis was $5.6 \%$ (4 of 71 patients; 3 Grade 3, and 1 Grade 5). The cumulative incidence of Grade $\geq 2$ radiation pneumonitis was $25 \%$. Other adverse events were as follows: Grade $\geq 2$ pleural effusion was detected in 2 patients; Grade 1 atelectasis was 1 patients; Grade $\geq 2$ dermatitis were observed in 4 patients; Grade 2 rib fractures were seen in 12 patients; and Grade 1 soft tissue swelling was detected in 2 patients, respectively.

Regarding the differences due to tumor location, the incidence of Grade $\geq 2$ radiation pneumonitis was $50 \%$ $(4 / 8)$ and $22 \%(14 / 63 ; p=0.09)$ for patients with a central tumor and those with a peripheral tumor, respectively. The incidence of Grade $\geq 3$ radiation pneumonitis was 13 and $4.7 \%$, respectively $(p=0.37)$. Other adverse events for central versus peripheral tumors were as follows: Grade $\geq 2$ pleural effusion, 0 versus $2(p=0.61)$; Grade 1 atelectasis, 0 versus $1(p=0.71)$; Grade $\geq 2$ dermatitis, 1 versus 3 ( $p=0.37$ ); Grade 2 rib fractures, 3 versus $9(p=0.10)$; and Grade 1 soft tissue swelling, 0 versus $2(p=0.61)$.

\section{Discussion}

In view of the poorer outcomes of stage IB NSCLC patients compared to those of stage IA patients, we used protocols to employ higher doses for larger tumors. Based on the toxicity results of the first study, we considered that the doses for $\mathrm{T} 1$ tumors could be further increased, and the total dose was escalated from 44 to 48 Gy for tumors $<1.5 \mathrm{~cm}$ and from 48 to 50 Gy for $1.5-3 \mathrm{~cm}$ tumors $[13,15]$. On the other hand, dose escalation was deferred for T2 tumors because Grade $\geq 2$ radiation pneumonitis was observed in $30 \%$ of $\mathrm{T} 2 \mathrm{a}$ 


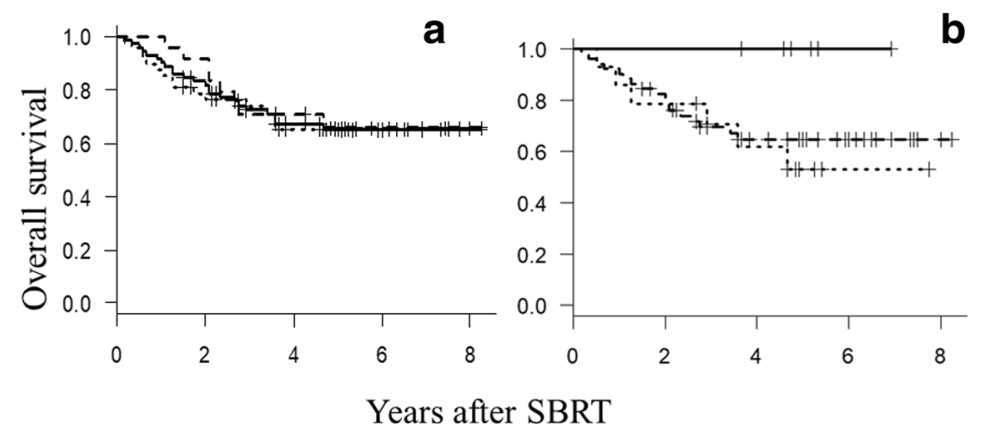

b

Number at risk

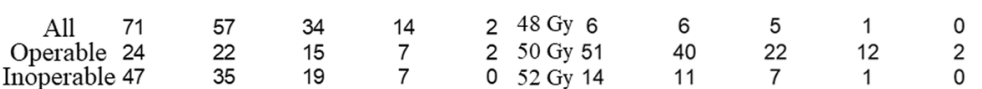

Fig. 1 a Overall survival curves for all patients (solid line), operable patients (dashed line) and inoperable patients (dotted line). b Overall survival curves for patients treated with 48 Gy/4 Fr (solid line), 50 Gy/4 Fr (dashed line), and 52 Gy/4 Fr (dotted line)

patients in the first study [13]. For T2a tumors, we planned to combine SBRT with S-1 chemotherapy, but the protocol was not approved by the institutional review board, so we used the same 52 Gy dose in the second study as in the first study. Therefore, the possible advantages of the second protocol were dose escalation by 2 to 4 Gy and the use of AAA for T1a/b tumors and the latter alone for T2a tumors. Pencil beam algorithm tends to overestimate the dose distribution in PTV, and AAA can provide more accurate dose distributions $[19,20]$. Using AAA could actually lead to higher dose delivery, and the isocenter dose is usually allowed to be considerably higher in order to deliver as low doses as possible to surrounding structures [21]. On the other hand, our isocenter dose prescription method that recommended to cover $95 \%$ of the PTV by at least $90 \%$ of the isocenter dose might lead to unnecessary dose spread to the surrounding tissues [22].

Although we attempted at dose escalation in the present study, other groups are using still higher doses. In a recent Japanese Clinical Oncology Group study 0702, dose escalation was attempted to determine the recommended dose for T2N0M0 patients [23]. The continual reassessment method was used, and the recommended dose was determined to be 55 Gy in 4 fractions as a dose covering $95 \%$ of the PTV for tumors $<100 \mathrm{~cm}^{3}$. The maximum and the isocenter doses were both $66.8 \mathrm{~Gy}$ in a patient with a prescribed dose of 55 Gy. In other studies from Western countries, 54 or 60 Gy in 3 fractions as the dose covering $95 \%$ of the PTV or to the $80 \%$ isodose line was used for peripheral

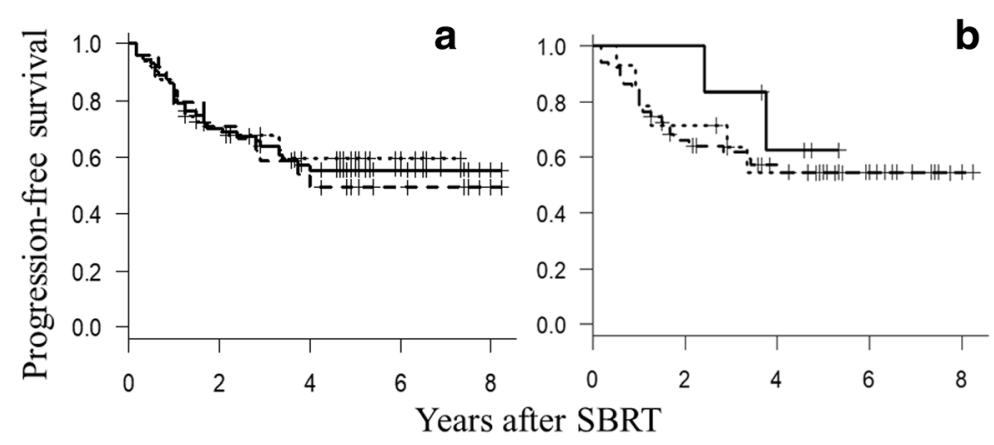

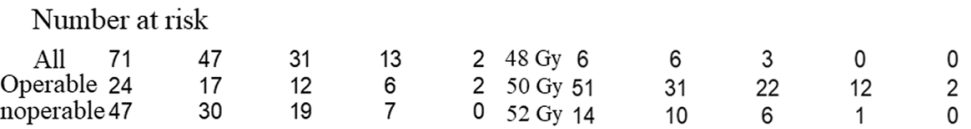

Fig. 2 a Progression-free survival curves for all patients (solid line), operable patients (dashed line) and inoperable patients (dotted line). b Progression-free survival curves for patients treated with $48 \mathrm{~Gy} / 4 \mathrm{Fr}$ (solid line), $50 \mathrm{~Gy} / 4 \mathrm{Fr}$ (dashed line), and $52 \mathrm{~Gy} / 4 \mathrm{Fr}$ (dotted line) 


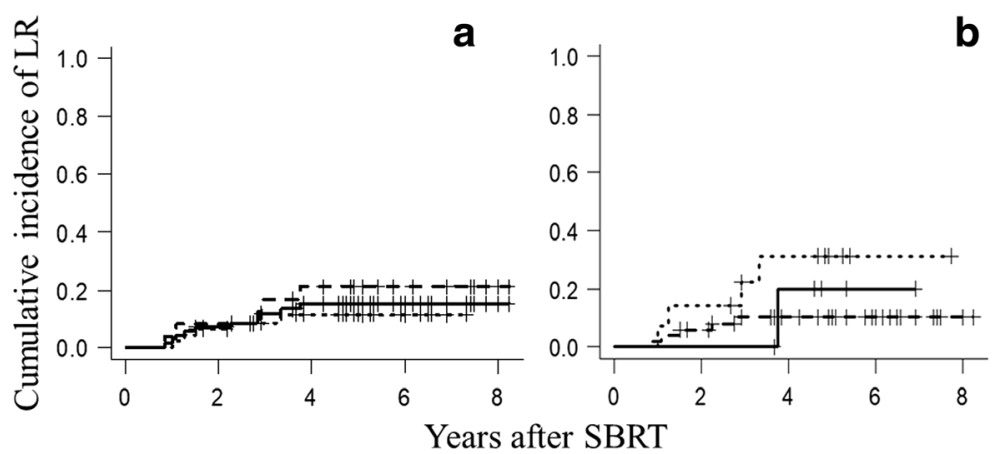

\section{b}

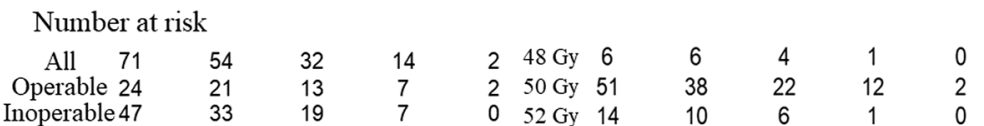

Fig. 3 a Cumulative incidences of local recurrence (LR) for all patients (solid line), operable patients (dashed line) and inoperable patients (dotted line). b Cumulative incidences of local recurrence for patients treated with $48 \mathrm{~Gy} / 4 \mathrm{Fr}$ (solid line), $50 \mathrm{~Gy} / 4 \mathrm{Fr}$ (dashed line), and $52 \mathrm{~Gy} / 4 \mathrm{Fr}$ (dotted line)

tumors, while slightly lower doses were prescribed for centrally-located tumors, T2 tumors, and tumors with chest wall invasion; all of them reported acceptable toxicities and favorable outcomes [6, 9, 24]. Since we prescribed the doses to the isocenter, the doses covering $95 \%$ of the PTV and doses to the $80 \%$ isodose line were still lower; our doses covering 95\% of the PTV were $89.7 \% \pm 2.8 \%$ (mean \pm standard deviation) of the isocenter dose, and the doses to the $80 \%$ isodose line were about $80 \%$ of the isocenter dose. Therefore, further dose escalation should be considered in future investigations.

In attempting at dose escalation, the relatively high incidence of Grade $\geq 2$ radiation pneumonitis may be a problem. However, one reason for the high incidence may be that all radiation pneumonitis events to which corticosteroids were prescribed by attending physicians were regarded as Grade 2 toxicity; in some cases, steroids might have been unnecessary. Another reason may be that our method to control respiratory tumor motion was not sufficient, since we only used abdominal compression and shallow breathing. Hence, the ITV margins became larger. In more recent patients not included in this analysis, we have used a breath-holding method, so the ITV margins have become smaller. Nevertheless, in general, complication rates in the present study were not greatly different from those of the first study $[13,15]$.
Grade $\geq 2$ radiation pneumonitis and rib fractures in patients with a tumor adjacent to the chest wall can develop at certain rates, so it may be difficult to decrease them. The incidence of Grade $\geq 2$ rib fracture was indeed higher in the present study $[13,15]$. On the other hand, skin, and esophageal toxicities may be avoided by taking care not to produce hot spots. Although there were no differences in toxicity between centrally located and peripheral tumors in this study, several studies suggest that SBRT for a central lesion increases a risk for severe radiation injury of normal tissues, such as the lung, large airways, great vessels, esophagus and heart $[16,24,25]$. So, larger fraction numbers may generally be recommended for central tumors.

To evaluate efficacy of the newer protocol, we reanalyzed 113 patients enrolled in our first study at our own institution thereby updating the follow-up data. In the first study, the median follow-up period was 51 months for all patients and 73.5 months for living patients. At 5 years, OS was $53 \%$ in the first study, while it was $65 \%$ in the present study $(p=0.18)$. PFS was $44 \%$ vs $55 \%$ ( $p=$ 0.09 ), and the cumulative incidence of LR was $15 \%$ in both studies $(p=0.82)$. Although OS and PFS at 5 years and later tended to be higher in the second than in the first study, there were no differences. The curves for the second study tended to lie above (data not shown), and the lack of significance may be due to the patient

Table 2 Three and 5-year data in all, operable, and inoperable patients

\begin{tabular}{lllll}
\hline & All patients $(n=71)$ & Operable patients $(n=24)$ & Inoperable patients $(n=47)$ & $p$-value ${ }^{\text {a }}$ \\
\hline OS (\%) at 3/5 years & $73 / 65$ & $71 / 66$ & $74 / 65$ & 0.78 \\
PFS (\%) at 3/5 years & $64 / 55$ & $58 / 49$ & $68 / 60$ & 0.61 \\
Cumulative incidence of LR (\%) at 3/5 years & $12 / 15$ & $17 / 21$ & $9 / 12$ & 0.34 \\
\hline
\end{tabular}

OS Overall survival, PFS Progression-free survival, $L R$ Local recurrence

${ }^{\text {a } D i f f e r e n c e ~ b e t w e e n ~ o p e r a b l e ~ a n d ~ i n o p e r a b l e ~ p a t i e n t s ~}$ 
Table 3 Three and 5-year data in patients receiving 48 Gy/4 Fr, 50 Gy/4 Fr, and 52 Gy/4 Fr

\begin{tabular}{llll}
\hline & $48 \mathrm{~Gy} / 4 \mathrm{Fr}(n=6)$ & $50 \mathrm{~Gy} / 4 \mathrm{Fr}(n=51)$ & $52 \mathrm{~Gy} / 4 \mathrm{Fr}(n=14)$ \\
\hline OS (\%) at 3/5 years & $100 / 100$ & $70 / 65$ & $71 / 53$ \\
PFS (\%) at 3/5 years & $83 / 63$ & $62 / 55$ & $64 / 54$ \\
Cumulative incidence of LR (\%) at 3/5 years & $0 / 20$ & $10 / 10$ & $22 / 31$ \\
\hline
\end{tabular}

OS Overall survival, PFS Progression free survival, $L R$ Local recurrence

number. If more patients are included in the second study, the differences may become significant. In this study, the OS, PFS, and cumulative incidence of LR did not differ between the operable and inoperable patients. So, the beneficial effect of SBRT especially in inoperable patients seems remarkable. Conventional radiotherapy yielded 5-year survival rates of $30 \%$ or lower in stage I NSCLC patients [26], so the advent of SBRT is quite valuable for these patients.

OS, PFS, and LR rates were also not greatly different between stage IA and stage IB patients. However, the LR rate for stage IB tended to be higher. If more patients are included, the differences may become significant. Nevertheless, improvement in treatment outcome may be relatively small, if any, even if we continue to use this second protocol. Improvement in local control is desirable for T2a tumors, and to achieve this, further dose escalation should be attempted. The use of more fraction numbers or particle therapy may be recommended $[27,28]$, but even when these policies are adopted, dose escalation should be investigated [29].

There are several limitations for this study. First, the sample size was relatively small; many neighboring hospitals started SBRT, and patient accrual slowed down. Second, low doses were used compared to other recent studies in the literature. Third, the high rate for Grade $\geq 2$ pneumonitis may not reflect the actual rate due to the early administration of steroids. Controlling the steroid administration may be necessary in future clinical studies.

\section{Conclusion}

The present study yielded favorable outcomes. However, escalating the total dose did not lead to improved outcomes, although OS and PFS at 5 years and later tended to be higher in the present than in the first study. Further dose escalation should be investigated in future studies.

\footnotetext{
Abbreviations

AAA: Analytical anisotropic algorithm; CT: Computed tomography; FDGPET: ${ }^{18}$ F-fluoro-deoxyglucose-positron emission tomography; Gy: Gray; ITV: Internal target volume; LR: Local recurrence; MV: Mega voltage; NSCLC: Non-small cell lung cancer; OS: Overall survival; PFS: Progression-free survival; PS: Performance status; PTV: Planning target volume; SBRT: Stereotactic body radiotherapy
}

\section{Acknowledgments}

We are grateful to Drs. Ryuji Asai, Hiroyuki Ogino, Rumi Murata, Chisa Hashizume, Shiho Ayakawa, Aiko Nagai, Hiromitsu Iwata, Shinya Otsuka, Katsura Kosaki, Shinya Takemoto, Michio Iwabuchi and Ran Takenaka for their contribution in collecting data.

\section{Funding}

The study was not funded.

\section{Availability of data and materials}

The clinical dataset supporting the conclusions of this article is available in the results section (Table 1). The raw data is provided in the Department of Radiology, Nagoya City University Graduate School of Medical Sciences.

\section{Authors' contributions}

AM carried out the study and drafted the manuscript. YS designed the study and gave final approval for publication. FB participated in the design of the study and helped to perform the statistical analyses. YM, TM, CS, TY and TT participated in the data acquisition and analysis. All authors have read and approved the final manuscript.

\section{Ethics approval and consent to participate}

The study protocol was approved by the institutional review board of Nagoya City University Graduate School of Medical Sciences (NCU-0803). Informed consent was obtained from all patients.

\section{Consent for publication}

Not applicable.

\section{Competing interests}

The authors declare that they have no competing interests.

\section{Publisher's Note}

Springer Nature remains neutral with regard to jurisdictional claims in published maps and institutional affiliations.

\section{Author details}

'Department of Radiology, Nagoya City University Graduate School of Medical Sciences, 1 Kawasumi, Mizuho-cho, Mizuho-ku, Nagoya 467-8601, Japan. ${ }^{2}$ Department of Radiology, Nagoya City West Medical Center, 1-1-1, Hirate-cho, Kita-ku, Nagoya 462-8508, Japan.

Received: 27 June 2017 Accepted: 5 September 2017

Published online: 11 September 2017

References

1. Baker S, Dahele M, Lagerwaard FJ, Senan S. A critical review of recent developments in radiotherapy for non-small cell lung cancer. Radiat Oncol. 2016;11:115

2. Ricco A, Davis J, Rate W, Yang J, Perry D, Pablo J, et al. Lung metastases treated with stereotactic body radiotherapy: the RSSearch ${ }^{\circledR}$ patient Registry's experience. Radiat Oncol. 2017;12:35.

3. Nagata Y, Negoro Y, Aoki T, Mizowaki T, Takayama K, Kokubo M, et al. Clinical outcomes of 3-D conformal hypofractionated single high dose radiotherapy for one or two lung tumors using a stereotactic body frame. Int J Radiat Oncol Biol Phys. 2002;52(4):1041-6.

4. Baumann P, Nyman J, Hoyer M, Wennberg B, Gagliardi G, Lax I, et al. Outcome in a prospective phase II trial of medically inoperable stage I non-small-cell lung cancer patients treated with stereotactic body radiotherapy. J Clin Oncol. 2009;27(20):3290-6. 
5. Takeda A, Sanuki N, Kunieda E, Ohashi T, Oku Y, Takeda T, et al. Stereotactic body radiotherapy for primary lung cancer at a dose of 50 Gy total in five fractions to the periphery of the planning target volume calculated using a superposition algorithm. Int J Radiat Oncol Biol Phys. 2009;73(2):442-8.

6. Timmerman R, Paulus R, Galvin J, Michalski J, Straube W, Bradley J, et al. Stereotactic body radiation therapy for inoperable early stage lung cancer. JAMA. 2010;303(11):1070-6

7. Onishi H, Araki T. Stereotactic body radiation therapy for stage I non-smallcell lung cancer: a historical overview of clinical studies. Jpn J Clin Oncol. 2013;43(4):345-50.

8. Guckenberger M. Stereotactic body radiotherapy for stage I NSCLC: the challenge of evidence-based medicine. J Thorac Oncol. 2014;9(2):e17-8.

9. Chang JY, Senan S, Paul MA, Mehran RJ, Louie AV, Balter P, et al. Stereotactic ablative radiotherapy versus lobectomy for operable stage I non-small-cell lung cancer: a pooled analysis of two randomised trials. Lancet Oncol. 2015;16(6):630-7.

10. Nagata Y, Hiraoka M, Shibata T, Onishi H, Kokubo M, Karasawa K, et al Prospective trial of stereotactic body radiation therapy for both operable and inoperable T1N0M0 non-small cell lung cancer: Japan clinical oncology group study JCOG0403. Int J Radiat Oncol Biol Phys. 2015:93(5):989-96.

11. Sun B, Brooks ED, Komaki RU, Liao Z, Jeter MD, McAleer MF, et al. 7-Year follow-up after stereotactic ablative radiotherapy for patients with stage I non-small cell lung cancer: results of a phase 2 clinical trial. Cancer. 2017. doi:10.1002/cncr.30693. Epub ahead of print.

12. Aoki M, Hatayama Y, Kawaguchi H, Hirose K, Sato M, Akimoto H, et al. Clinical outcome of stereotactic body radiotherapy for primary and oligometastatic lung tumors: a single institutional study with almost uniform dose with different five treatment schedules. Radiat Oncol. 2016;11:5.

13. Baba F, Shibamoto $Y$, Ogino $H$, Murata $R$, Sugie $C$, Iwata $H$, et al. Clinical outcomes of stereotactic body radiotherapy for stage I non-small cell lung cancer using different doses depending on tumor size. Radiat Oncol. 2010;5:81

14. Shibamoto Y, Hashizume C, Baba F, Ayakawa S, Manabe Y, Nagai A, et al. Stereotactic body radiotherapy using a radiobiology-based regimen for stage I nonsmall cell lung cancer. A multicenter study. Cancer. 2012;118(8):2078-84.

15. Shibamoto Y, Hashizume C, Baba F, Ayakawa S, Miyakawa A, Murai T, et al. Stereotactic body radiotherapy using a radiobiology-based regimen for stage I non-small-cell lung cancer: five-year mature results. J Thorac Oncol. 2015;10(6):960-4.

16. Timmermann R, McGarry R, Yiannoutsos C, Papiez L, Tudor K, DeLuca J, et al. Excessive toxicity when treating central tumors in a phase II study of stereotactic body radiation therapy for medically inoperable early-stage lung cancer. J Clin Oncol. 2006;24(30):4833-9.

17. Baba F, Shibamoto Y, Tomita N, Ikeya-Hashizume C, Oda K, Ayakawa S, et al. Stereotactic body radiotherapy for stage I lung cancer and small lung metastasis: evaluation of an immobilization system for suppression of respiratory tumor movement and preliminary results. Radiat Oncol. 2009;4:15.

18. Takenaka R, Shibamoto Y, Miyakawa A, Hashizume C, Baba F. The fate of residual tumor masses that persist after stereotactic body radiotherapy for solitary lung nodules: will they recur? Clin Lung Cancer. 2016;17(5):406-11.

19. Herman Tde L, Hibbitts K, Herman T, Ahmad S. Evaluation of pencil beam convolution and anisotropic analytical algorithms in stereotactic lung irradiation. J Med Phys. 2011;36(4):234-8.

20. Badkul R, Nicolai W, Pokhrel D, Ramanjappa T, Jiang H, Wang F, et al. SU-FT-622: comparative analysis of pencil beam and anisotropic analytical algorithm (AAA) for stereotactic body radiation therapy (SBRT) of thoracic spine. Med Phys. 2016;43(6):3607.

21. Suzuki O, Mitsuyoshi T, Miyazaki M, Teshima T, Nishiyama K, Ubbels JF, et al. Dose-volume-response analysis in stereotactic radiotherapy for early lung cancer. Radiother Oncol. 2014;112(2):262-6.

22. Widder J, Hollander M, Ubbels JF, Bolt RA. Langendijk. Optimizing dose prescription in stereotactic body radiotherapy for lung tumours using Monte Carlo dose calculation. Radiother Oncol. 2010;94(1):42-6.

23. Onimaru R, Onishi H, Shibata T, Hiraoka M, Ishikura S, Karasawa K, et al. Phase I study of stereotactic body radiation therapy for peripheral T2NOMO non-small cell lung cancer (JCOG0702): results for the group with PTV $\geqslant 100 c c$. Radiother Oncol. 2017;122(2):281-5.

24. Senthi S, Lagerwaard FJ, Haasbeek CJ, Slotman BJ, Senan S. Patterns of disease recurrence after stereotactic ablative radiotherapy for early stage non-small-cell lung cancer: a retrospective analysis. Lancet Oncol. 2012;13(8):802-9.
25. Rowe BP, Boffa DJ, Wilson LD, Kim AW, Detterbeck FC, Decker RH. Stereotactic body radiotherapy for central lung tumors. J Thorac Oncol. 2012;7(9):1394-9.

26. Jeremic B, Shibamoto Y, Acimovic L, Milisavljevic S. Hyperfractionated radiotherapy alone for clinical stage I nonsmall cell lung cancer. Int J Radiat Oncol Biol Phys. 1997;38(3):521-5.

27. Haasbeek CJ, Lagerwaard FJ, Slotman BJ, Senan S. Outcomes of stereotactic ablative radiotherapy for centrally located early-stage lung cancer. J Thorac Oncol. 2011;6(12):2036-43.

28. Shibamoto Y, Miyakawa A, Otsuka S, Iwata H. Radiobiology of hypofractionated stereotactic radiotherapy: what are the optimal fractionation schedules? J Radiat Res. 2016;57(Suppl 1):i76-82

29. Shibamoto Y. Particle therapy: a suitable alternative to stereotactic body radiotherapy for stage I non-small-cell lung cancer? Lung Cancer Manage. 2013;2(5):353-6.

\section{Submit your next manuscript to BioMed Central and we will help you at every step:}

- We accept pre-submission inquiries

- Our selector tool helps you to find the most relevant journal

- We provide round the clock customer support

- Convenient online submission

- Thorough peer review

- Inclusion in PubMed and all major indexing services

- Maximum visibility for your research

Submit your manuscript at www.biomedcentral.com/submit

) Biomed Central 